\title{
Effect of Motor Vehicle Structural Vibration on Human Body - Review
}

\author{
Pranavdeep A. Borse ${ }^{1}$, Dr. Purushottam. S. Desale ${ }^{2}$ \\ PG Student, Mechanical Engineering Department, S.S.V.P.S.B.S. Deore College of Engineering, Dhule, India ${ }^{1}$ \\ Associate Professor, Mechanical Engineering Department, S.S.V.P.S.B.S. Deore College of Engineering, Dhule, India ${ }^{2}$
}

\begin{abstract}
As India is the second largest manufacturer of two wheeler Motorcycles, so most of the Indian population prefer two wheelers for their daily travelling. There is also some reasons like economic, travelling distance and traffic on city roads behind preference given to two wheelers. During travelling on two wheelers many parts of body are directly in contact with vehicle but Hands are main interface between rider and two wheeler. While travelling, handlebar transfers vehicles vibration to human body. These vibrations are due to engines inertia and combustion force, road induced vibration \& improper structural designs and they are quiet varying with respect to speed, fuel supply. The prolonged exposure to these vibration may affect different parts of human body mostly hands, spine, lower back. This exposure to part of body of operator is called as 'Segmental vibration' which will result in 'hand-arm vibration syndrome' (HAVS). These vibrations also results in mental retardness and high blood pressure which are very severe. This paper is the review the work in the area of HAVS and reveals the fact that, up to certain limit vibration are not hazardous but after exceeding that limit they are very hazardous.
\end{abstract}

Keywords: Motorcycles, vibration, Hand Arm Vibration, Whole Body Vibration, Handlebar.

\section{INTRODUCTION}

The IC engine is the source of power in automobiles which is mounted on the skeleton of motorvehicle. If it is not mounted properly it will start vibrating and those will be transferred to the mounting, chessis and start affecting drivability, comfort, driving stability. This hazardous vibration must have to be reduced to take care of human being as per market survey, customer / user requirements. IC engine's vibration behaviour depends on unbalanced rotating and reciprocating parts, cyclic variation in gas pressure, shaking forces of reciprocating parts and structural design of the engine mounts. Engine vibrations are caused due to the reciprocating and rotating masses of the engine. The motorcycle gives different kinds of vibrations to its user from vibrations of it engine, rotating and reciprocating parts of engine, imperfect structural design of the two wheeler and bad road conditions. It is observed that these vibrations are very hazardous to the health, if it exceeds beyond permissible limit it may cause the illness of spine, musculoskeletal symptom in the lower back as well as the neck and upper limbs. Experimental studies in different literatures on the transmission of vertical vibration show that vibration affect health of motorcycle rider if exceed certain limit. It is therefore necessary to develop suitable guidelines for manufacturing of two wheelers by investigating influence of vibration to motorcycle rider. The severity of these harmful vibrations is reduced by providing a standard type of modification in handlebar, seat, front and rear suspension.

\section{Hand arm vibration and their effects}

The Hand arm vibration (HAV) is vibration which transmitted from handheld equipment like jack hammers, handle bar and steering wheel into the hands and arms of operators. It leads to vibration induced white finger (VWF).
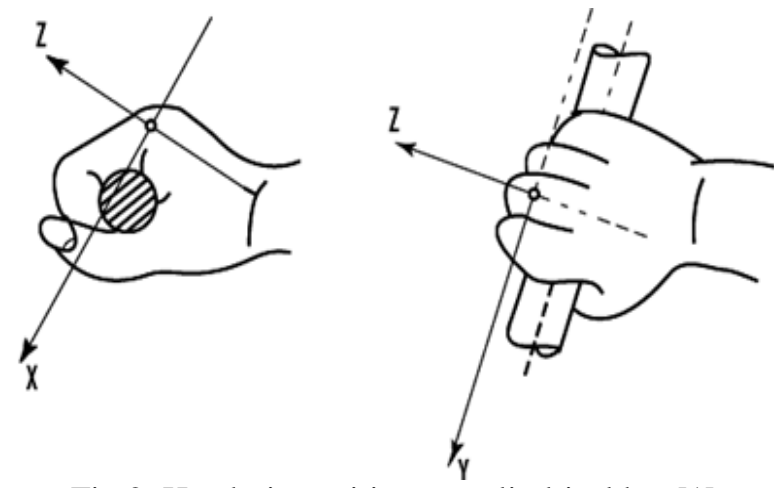

Fig 2: Handgrip position on cylindrical bar.[1] 
This at primary stage. If not, it can lead to permanent disability in the hands usefulness. Steering wheel vibration levels about $1 \mathrm{~m} / \mathrm{s} 2$ have been reported in one study. HAV at this level may present a slight risk of injury considering the long duration exposure while driving. It had also found that the rates of finger stiffness, finger numbness, and shoulder pain and shoulder stiffness were observed significantly higher among traffic motorcyclists as compared with the control group.

\section{Whole Body Vibration and their Effects}

The Whole body vibration (WBV) occurs when occupant sit or stand on vibrating seats or foot pedals. Prolonged exposure to higher vibration levels of WBV causes motion sickness, fatigue and headaches. WBV is one of the strongest factors of risk for low back disorders.

In typical whole-body vibration exposure levels of heavy vehicle drivers are in the range 0.4 to $2.0 \mathrm{~m} / \mathrm{s} 2$. Vibration found highest in the frequency range 2 to $4 \mathrm{~Hz}$. For a seated person vibration in the range of 4 to $8 \mathrm{~Hz}$ cause the entire upper torso to resonate and should be reduced and avoided. Vibrations with less than $0.315 \mathrm{~m} / \mathrm{s} 2$ are found to be comfortable, in between $0.315 \mathrm{~m} / \mathrm{s} 2$ and $2.5 \mathrm{~m} / \mathrm{s} 2$ are found to be uncomfortable and greater than $2.5 \mathrm{~m} / \mathrm{s} 2$ are found to be extremely uncomfortable. Health effects that associated with WBV and especially the driving environment are kidney disorders, high blood pressure, piles and impotence.

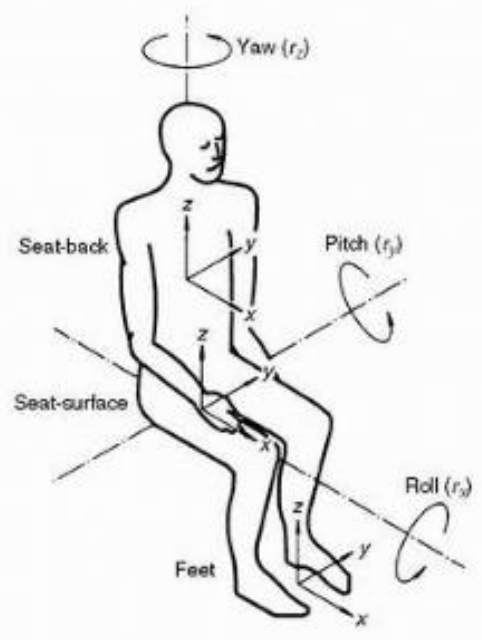

Seated position

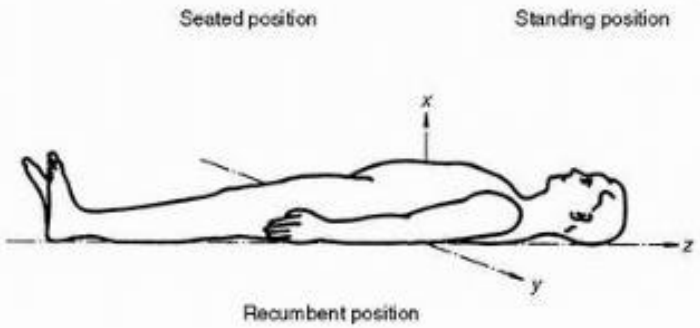

Fig 2: Basicentric axes of Human body [2]

\section{LITERATURE REVIEW}

Harale Shivraj. N Gyanendra Roy [3] describe in this analysis the handle bar assembly is excited with acceleration derived from road load data over range of 0 to $200 \mathrm{~Hz}$ which is an operational frequency to evaluate the strength of mountings on handle-bar in vibration. Using Altair solver code Bulk data Frequency response analysis on handle bar assembly is carried out. In this analysis the handle bar assembly is excited with dynamic loads i.e. acceleration derived from road load data for an operational frequency range to evaluate the strength of mountings on handle-bar in vibration. Pre-processing of Model preparation done using Hyper Mesh and Post processing is done using Hyper View and Hyper Graph. The simulation results for Mirror mounting bracket and Headlamp mounting casing are taken which are correlated with the experimental results in which failure location and pattern is exactly matched. Further design modifications have been incorporated for Mirror mounting bracket and Headlamp mounting casing to meet the strength requirement. 
Rebecca Wolfgang, et al. [4] studied that Long term exposure to WBV is a recognized risk factor for degenerative changes in the spine, subsequent back pain, and other adverse health effects. The haul truck drivers working at surface mines are exposed to whole-body vibration for prolonged periods. Thirty-two whole-body vibration (WBV) measurements were gathered from haul trucks under a range of normal operating conditions. Measurements taken from 30 of the 32 trucks fell within the health guidance caution zone defined by ISO2631-1 for an 8 hours exposure daily. This daily exposure increases the risk of long term health effects, and particularly back injury.

Systematic WBV measurements taken by mine sites at frequent intervals are required to provide an understanding of the causes of elevated vibration levels and hence determine appropriate control measures. This may be facilitated by the availability of accelerometers within consumer electronic products

Jaimon Dennis Quadros, et al. [5] studied, analyzed and obtain the idealized operating conditions of the human body. Prolonged exposure to specific frequencies of vibration might have effects on certain segment of the body. Their analysis has shown that for the given test vehicle and human body model, the ideal operating speed for HERO HONDA SPLENDOR vehicle on the terrain of specified amplitude for given input is found about $49.66 \mathrm{kmph}$

S. Agostoni et al. [6] research work has developed with the aim of investigating motorcycles ride comfort. Particular attention was focused on the handlebar, because this component is directly comes in contact with driver. A wellgrounded, repeatable methodology is developed with a proposed view of designing a Tuned Mass Damper (TMD) that should capable of absorbing

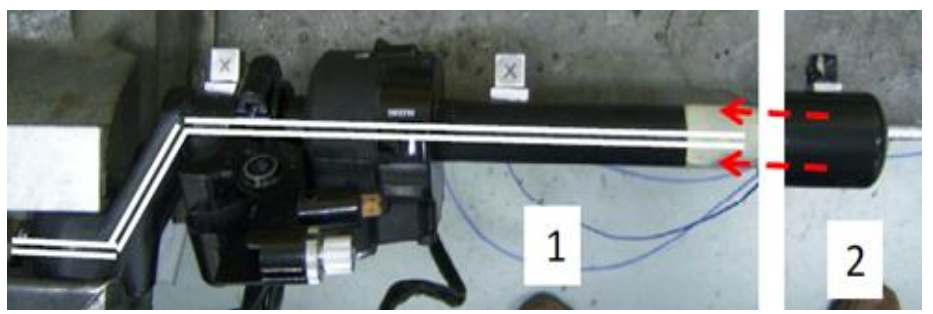

Fig 3: Handlebar: (1) Fixed beam (2) Insertion of steering balance into handle bar extremity [6]

vibrations, i.e. one which is capable of reducing the vibrations transmitted to the driver via the handlebar. The steering balances which placed at the extreme ends of the handlebar of all regular motorcycles is the starting point chosen for this methodology. Structural vibrations reduction is also mean of this paper. Thus, not only reduced driver exposure to engine unbalance vibration, but also improved ride comfort.

Yuichi Sugita, Junji Yoshida, Yusuke Itahashi.,[7] studied the engine input force to reduce a brush cutter handle vibration. Then modification in centrifugal clutch structure to control the input force done without changing the engine structure itself. In beginning, vibration of a brush cutter was measured and main factor of the large vibration was considered. Result showed that the large first order vibration generated by the inertia of the crank-piston system was found to be the main factor. Subsequently, at left handle of the brush cutter operational TPA was applied to find out the input force main contributing direction to the handle i.e. horizontal or vertical. As the result, it was clarified that the engine input force along the horizontal direction mainly affecting the handle vibration than the force along the vertical direction. Then, approach towards reduce the horizontal input force by using the unbalancing clutch to reduce the handle vibration has tried. For the reduction of horizontal axis input force, the small weight of $6 \mathrm{~g}$ was attached at opposite side of the crank-shaft counterweight and investigation through the operational test for the influence of the unbalancing clutch was done. The result indicated that by applying the weight for unbalancing by considering the contribution of the input force in each direction, brush cutter handle vibration could be reduced very effectively.

Gourav.p.sinha, p.s.bajaj [8] examined about the practical measurement of vibration occurring on two wheeler vehicle which are very dangerous when it is transmitted to human body through thigh, footrest, seat \&handle. So determining the vibration level occurring in vehicle should be helpful and some steps to reduce it are taken. In this paper from vibration point of view different aspect of riding vehicle on smooth and uneven road they will come to know that.

Xueyan S et al. [9] studied with objective to evaluate an adapter method for measuring the human arms vibration. Four different weight instrumented adapters were used to measure transmitted vibration to the wrist, forearm, and upper arm of each subject. At each location on the subjects separate adapter was attached using an elastic cloth wrap. For evaluating the validity of the adapter method two laser vibrometers were also used to measure the transmitted vibration at each location. This study found that with some systematic differences the adapter and laser-measured transmissibility spectra were comparable. 
Table 1-Reactions of Persons to Whole-Body Vibration Expressed in Terms of the Overall Vibration Value [2]

\begin{tabular}{|l|l|}
\hline \multicolumn{2}{|c|}{ Vibration Reaction } \\
\hline Less than $0.315 \mathrm{~m} / \mathrm{s} 2$ & Not uncomfortable \\
\hline 0.315 to $0.63 \mathrm{~m} / \mathrm{s} 2$ & A little uncomfortable \\
\hline 0.5 to $1 \mathrm{~m} / \mathrm{s} 2$ & Fairly uncomfortable \\
\hline 0.8 to $1.6 \mathrm{~m} / \mathrm{s} 2$ & Uncomfortable \\
\hline 1.25 to $2.5 \mathrm{~m} / \mathrm{s} 2$ & Very uncomfortable \\
\hline Greater than $2 \mathrm{~m} / \mathrm{s} 2$ & Extremely uncomfortable \\
\hline
\end{tabular}

H. Ashrafiuon et al. [10] focused on frequency response of an aircraft engine to determine harmonic forces. The locations, orientations, and types of mounts used are all critical in minimizing the transmitted forces. They also presented the results for a specific aircraft engine. The methodology of this work was applicable to most of the vibration isolation systems.

Mohsen Fereydooni1, Ali Nejat Lorestani, Hekmat Rabbani, Payam Javadikia [11] In this study, object is to compare effect of change engine rotation and ground type on operator of tractors and implements that utilized. In other words, the goal is to measurement and analysis of transmitted vibration on different parts of human body. In this investigation universal tractor and ferguson285 \&299 tractors with moldboard plough and disk are used. Hand-Arm vibration's operator in 1300, 1500 and $1700 \mathrm{rpm}$ and in ploughing field and unploughing field with hand-arm vibration meter are measured. After statistical analysis, appeared that effective vibration difference on hand and arm's operator in examined tractors is significant and engine rotation is significant too. Based on the results of statistical analysis, we can conclude that at the same terms the lowest vibration occurs respectively working with Massey Ferguson 285, 299 and Universal 650 tractors. Disking operator exposure to less vibration than he worked with moldboard plough and operator exposure to less vibration on plough ground than he worked on unplough ground. However increasing the engine rotation will increase vibrations on operator.

\section{III.CONCLUSION}

Motorcycle vibration is caused mainly due to Engine unbalanced force vibration and due to road induced vibration. As the Indian population mostly prefers motorcycle for daily use due to reasons like economic, road conditions, travelling distance etc. this regular prolonged exposure of human body to vibration may cause different types of syndrome like back injury, spondelysis, shoulder pain, hand induced white finger, drawiness, motion sickness, etc. As the vibrations transmitted to the rider through touch points like thigh, footrest, seat \&handle are of two types like Hand Arm Vibration (HAV) and Whole Body Vibration (WBV).

Following are the observations from the above literature review:

1. Vibration analysis of handlebar assembly is done for $20-200 \mathrm{~Hz}$ and then structural modifications in the mirror mounting bracket and headlamp mounting has been done by doing strength analysis.

2. Survey of vibration effect on haul truck drivers has made and found that for WBV daily exposure of 8 hours results in health hazards.

3. By Designing mathematical model for motorcycle rider found that for ideal operating speed for Splendor vehicle is $49.66 \mathrm{~km} / \mathrm{h}$.

4. Tuned mass damper having 5 degree of freedom has designed in order to reduce handlebar vibration and increase ride comfort. The mass of damper must be equal to $10 \%$ of modal mass of handle.

5. Counter weight attached to the engine crank shaft resulted in reduction of horizontal unbalanced force and there by reduction of brush cutter handle vibration.

6. HAV analysis in case of different tractor done by varying engine rpm found that in disk operator exposed to less vibrations as compared to moldboard plough. Also increasing engine rpm will results in increase of operator's vibration.

7. International organization for standardization (ISO) also explains reaction of human body at different vibration levels.

It is observed that the research was mainly on hand arm vibration. Research was done in area of heavy machinery, mining industry, power tools, and brush cutter but there is need of research to be done to minimize Handle bar vibration in motor cycle as it is the main source in case of transfer of vibration to user's body. 
UGC Approved Journal

IARJSET

ISO 3297:2007 Certified

Vol. 4, Issue 7, July 2017

\section{REFERENCES}

[1] International Organization for Standardization ISO 5349-1, Mechanical vibration - Measurement and evaluation of human exposure to handtransmitted vibration - part 1: general requirements, 2001

[2] International Organization for Standardization ISO 2631-1, Mechanical vibration and shock - Evaluation of human exposure to whole-body vibration - part 1: general requirements, 1997.

[3] Harale Shivraj. N, Gyanendra Roy "Vibration Analysis of 2 Wheeler Handle-Bar Assembly" Mahindra 2 Wheelers Ltd. Mahindra 2 Wheelers Ltd. 2012, PP. 1-7.

[4] Rebecca Wolfgang, Robin Burgess-Limerick "Whole-body vibration exposure of haul truck drivers at a surface coal Mine" Elsevier Applied Ergonomics, 2014.

[5] Jaimon Dennis Quadros1, Suhas2, Vaishak N.L3, Shilpa.B, "Study of vibration and its effects on health of a two wheeler rider", International Journal of Research in Engineering and Technology Volume: 02 Issue: 08 | Aug-2013.

[6] S. Agostoni, A. Barbera, E. Leo, M. Pezzola, M. Vanali, Investigation on motorvehicle structural vibrations caused by engine unbalances (1st part-Handlebar),Proceedings of the SEM Annual Conference June 1-4, 2009 Albuquerque New Mexico USA @2009 Society for Experimental Mechanics Inc.

[7] Yuichi Sugita, Junji Yoshida, Yusuke Itahashi., "Handle Vibration Reduction of Brush Cutter By Unbalancing Centrifugal Clutch"23rd International Congress on Sound and Vibration, ICSV23, Athens (Greece), 10-14 July 2016.

[8] Gourav.P.Sinha, P.S.Bajaj "Vibration analysis of hero honda vehicle" IJMPE, Volume-2, Issue 2 Feb-2014.

[9] Xueyan S. Xu , Ren G. Dong, Daniel E. Welcome, Christopher Warren, Thomas W. McDowell, An examination of an adapter method for measuring the vibration transmitted to the human arms, Measurement 2015, 73, pp.318-334.

[10] Ashrafiuon, H., and Natraj, C (1992) Dynamic Analysis of Engine-Mount Systems, ASME J. Vibr.Acoust., 114 : 79-83

[11] Mohsen Fereydooni, Ali NejatLorestani, HekmatRabbani, PayamJavadikia. Measurement and Analysis of Vibration of Operator in Universal 650, Massey Ferguson 285 \& MF 299 Tractors. International journal of the mechanics and Applications 2012, 2(5): 88-92 DOI:10.5923/j.mechanics. 20120205.06 